\title{
Quantitative Analysis and Comparison of Diffusion Tensor Imaging Tractography Algorithms
}

\author{
Steve Crettenand ${ }^{\dagger}$, Stephen D. Meredith ${ }^{\dagger}$, Matthew J. Hoptman* and Richard B. Reilly ${ }^{\dagger}$ \\ ${ }^{\dagger}$ School of Electrical, Electronic \\ \&3 Mechanical Engineering \\ University College Dublin \\ IRELAND \\ * Division of Clinical Research \\ Nathan Kline Institute for Psychiatric Research \\ Orangeburg, New York \\ USA
}

E-mail: steve.crettenand@ee.ucd.ie

stephen.meredith@ee.ucd.ie Hoptman@NKI.RFMH.ORG

richard.reilly@ucd.ie

\begin{abstract}
This paper describes a platform developed for analysis of Diffusion Tensor Image data using different tractography algorithms. The platform allows these algorithms to be compared both quantitatively and qualitatively. Two specific tractography algorithms were compared, STT (Streamlines Tracking Technique) and TEND (tensor-deflection algorithm). The platform was assessed on a publicly available DTI dataset to analyse and quantify the performance of these tractography algorithms. Based on specific tests using this platform, results indicate that the STT algorithm is better at dealing with fibres containing curves and TEND is more appropriate for straighter fibres. A methodology is also proposed to help differentiate between nerve fibres that meet or cross.
\end{abstract}

Keywords - Diffusion Tensor Imaging (DTI), fibre tractography, STT, TEND

\section{INTRODUCTION}

Diffusion Tensor Imaging (DTI) (or Diffusion Tensor MRI) is an extension of MRI that quantifies water diffusion in living tissues [1], [2], [3] and [4]. DTI, like MRI, is a non-invasive method [5] which can be performed safely and repeatedly in vivo.

An important application of DTI is the ability to track nerve fibre pathways in the brain [6]. Such information could help the diagnosis of certain diseases like multiple sclerosis, schizophrenia [7] and autism. Therefore, DTI opens a window for understanding connectivity within the brain.

Diffusion can be understood as the dilatation or the propagation of a droplet on a surface. There are two types of diffusion: isotropic and anisotropic. Isotropic diffusion has no preferred direction, i.e. diffusion is equal in all directions. This is typical in grey matter. Anisotropic diffusion has diffusion in some directions more than others. This form of diffusion is typical in white matter. It has been reported that diffusion of water molecules is greatest parallel to the direction of nerve fibres [5].

Diffusion weighted gradients must be applied in multiple directions to obtain the information required to generate/calculate the diffusion tensor. The diffusion tensor is a $3 \times 3$ real valued symmetric matrix with 6 degrees of freedom. Diagonal terms represent molecular mobility along principal directions of diffusivity and off diagonal terms arise due to the fact that measurements are made in the reference frame of the scanner which is not typically the same as the diffusion frame of reference [5]. Gradients applied in at least 6 different directions are required in order to calculate the tensor.

Having obtained the diffusion tensor for each voxel, standard parameters such as fractional anisotropy (FA), a measure of diffusion anisotropy and eigenvalues/eigenvectors can be calculated. A voxel is the $3 \mathrm{D}$ equivalent of a pixel and is the smallest distinguishable unit of volume. The FA is scaled from 0 (isotropic) to 1 (anisotropic):

$F A=\sqrt{\frac{1}{2}} \frac{\sqrt{\left(\lambda_{1}-\lambda_{2}\right)^{2}+\left(\lambda_{2}-\lambda_{3}\right)^{2}+\left(\lambda_{3}-\lambda_{1}\right)^{2}}}{\sqrt{\lambda_{1}^{2}+\lambda_{2}^{2}+\lambda_{3}^{2}}}$

where $\lambda_{i}$ are the eigenvalues following diagonalization of the tensor.

While direct visualisation of the tensor information is not possible, methods exist to aid visual 


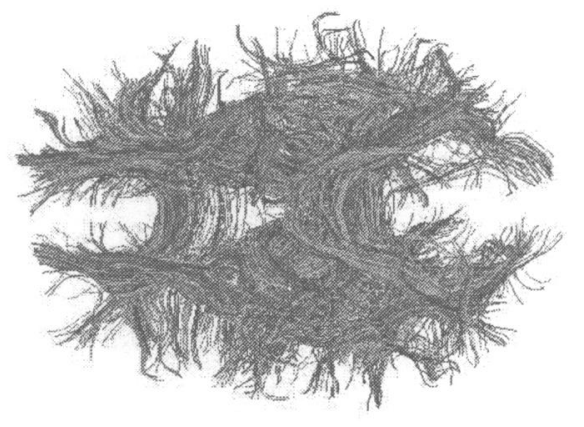

Fig. 1: Typical output from DTI analysis, showing connections in the brain using the STT algorithm. Image shows the axial plane with frontal lobes to the left and the occipital region to the right.

interpretation. These methods include anisotropy maps and colour maps. In anisotropy maps, the white matter appears brighter than the grey matter, and some fibre structures can be observed. Colour maps provide a colour coded visualisation of the direction of the principal eigenvector. Indeed the three main axes (right-left, anteriorposterior and superior-inferior) are assigned a determined colour: red (R), green $(\mathrm{G})$ and blue $(\mathrm{B})$. Therefore the major fibre orientation is drawn using a combination of these three colours and using the FA value to represent the signal intensity [8].

$3 \mathrm{D}$ visualisations can also be generated. One method consists of representing the tensor by ellipsoids. For an isotropic representation this produces a sphere and for the anisotropic representation produces a linear or planar ellipsoid oriented in the direction of its longest axis. A composite representation also exists [9]. The most intuitive $3 \mathrm{D}$ representations are based on fibre tractography algorithms [10], [11], [12] and [13]. These algorithms attempt to display the nerve fibres present in the brain based on diffusion tensor information.

Despite the importance of tractography and the number of proposed algorithms, few comparisons of these algorithms have been reported in the literature. One of the reasons for this is the lack of a common platform to allow qualitative and quantitative comparisons of these algorithms.

\section{AIM}

The aim of this study was to analyse and compare different DTI tractography algorithms based on a standard framework.

The platform developed (Fig. 2) was used to assess two tractography algorithms using a synthetic dataset and real data. The first standard tractography method assessed was the STT (Streamlines Tracking Technique) [14], [15] and [16]. This method follows the principal diffusion direction throughout the volume. This direction is given by the principal eigenvector which is assumed to be tangent to the pathway. The second algorithm assessed was the TEND (TENsor-Deflection) algorithm [9], [17] and [18], which uses the tensor at each point to multiply the incoming path vector, resulting in a new vector that is deflected toward the principal direction of diffusion.

$$
\mathbf{v}_{\text {out }}=\mathbf{D} \cdot \mathbf{v}_{\text {in }}
$$

The second goal of this research was to provide comparison measures to better understand the limitations of the fibre tractography algorithms and identify possible improvements. Given the recent generation of a publicly available synthetic DTI dataset [19], comparison testing can readily be carried out to fully understand how these algorithms work and allow selection of the best operating parameters.

Comparisons of tractography algorithms is of great scientific interest as it provides a framework to help better understand real data for the detection and study of brain disorders such as autism or schizophrenia. Real data for this study was provided by the Nathan Kline Institute for Psychiatric Research in New York.

Section III provides an explanation of the developed platform and of the synthetic dataset. Section IV presents the different analysis methods performed: a comparison between two tractography algorithms using a synthetic DTI dataset (subsection $a$ ), quantification and measures of performance for the two tractography algorithms (subsection $b$ ) and some results using real patient data (subsection $c$ ). In section $\mathrm{V}$ we discuss the results obtained and finally, section VI provides an outline of possible future work.

\section{Platform \& Database}

The developed platform accepts DTI data as an input and provides a format for analysis and visualisation. The framework combines five successive DTI processing steps, loading the data files, tensor computation, rewriting, converting and compressing. Pre-computing visualisation parameters and visualisation in this study were achieved using the DTI Query software [20]. This visualisation software was chosen as it uses dynamic queries to enable interactive manipulation of the fibre parameters and ROI positions, thus facilitating examination and analysis of data.

The Common DTI Dataset, named PISTE, was developed for researchers focused on DTI analysis [19] and allows researchers to test, validate and compare algorithms. It is a common dataset which offers a good point of comparison between different methods of tractography.

The dataset contains ten different shapes which represent typical fibres: linear, linear with a break, branching, three crossings (curve, straight and 


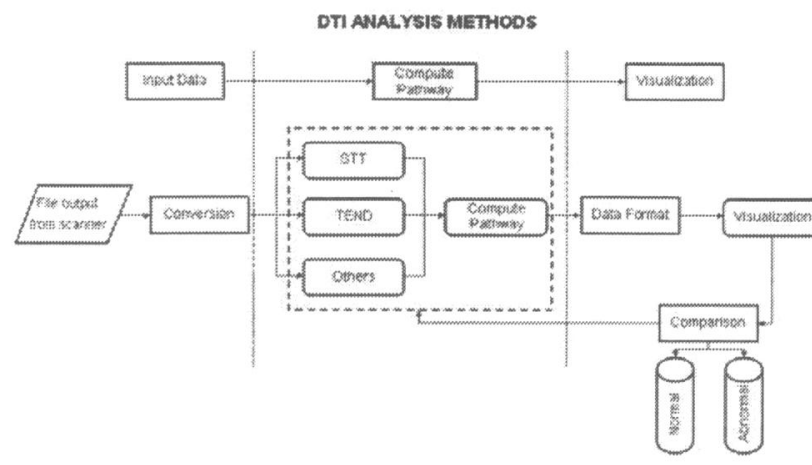

Fig. 2: An analysis and comparison platform for DTI tractography algorithms

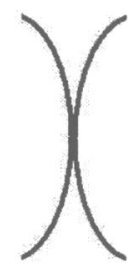

(a)

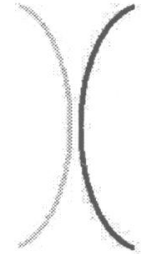

(b)

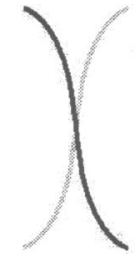

(c)
Fig. 3: Example of two different fibre shapes (a), kissing fibres (b) and curving fibres (c)

cross) and an example of kissing fibres (Fig. 3.b - fibres that meet but not cross). Other fibre examples which do not correspond to likely neural pathways in the brain such as spirals, helical structures and even a leaf are provided to further test the tractography algorithms.

\section{Analysis Methods}

\section{a) Interpretation of the Synthetic Dataset}

The Common DTI Dataset contains 10 different shapes, each one having three different SNR values $(7,15,30)$. In this dataset some shapes are more important and more interesting for medical analysis because they resemble nerve connections. These include linear, kissing, crossing and branching shapes. In this study attention is focused on these shapes.

For example, one of the most important medical imaging questions in DTI relates to the ability of tractography algorithms to distinguish kissing (Fig. 3.b) from curving fibres (Fig. 3.c) [21]. In changing the parameters of the visualisation: the minimum and the maximum value of the length, the FA and the average curvature of the fibres, the shapes were qualitatively analysed and in particular methods were determined to distinguish these two fibre types.

Noise From analysis of the dataset shapes it was observed that precision of tractography algorithms is a function of the noise present in the data. When using the TEND algorithm for shapes (particularly those with two branches) at low SNR values there was one preferred direction. This implies that one branch is better represented than the other. The STT algorithm normally does not produce perfectly straight pathways; some curvature is generally visible at the extremities. This is due to the fact that the STT algorithm follows the principal diffusion direction but also produces some errors as it takes into consideration noise around the fibre shape. Another explanation of the curvature is that an appropriate FA Termination Threshold (a key parameter of the pre-processing) was not selected. Therefore, the two algorithms are influenced by noise, but the TEND algorithm is more immune to the influence of noise around the shape and thus produces "cleaner" representations.

It was noted that STT is better at dealing with fibres containing curves [17] but not all types of curvature. However observing the result of the helical shape it was noticed that better results are obtained with the TEND algorithm due to the background noise. While we do not find a physical analogous fibre with a helical shape in the brain, it is interesting to observe the algorithmic behaviour on such a complex fibre structure. The acquired knowledge can be used, for example, to design new stopping criteria for tractography algorithms.

Length \& FA value The two major parameters that are adjusted to provide the best visualisation, the range of lengths and range of FA values of the pathway, were also examined. As our aim is to locate possible connections in the brain, by experimenting with the synthetic dataset it was found advantageous to simply increase the value of the minimum length of the pathway and then adjust the range of $\mathrm{FA}$ values to obtain our final estimate of the connection.

Kissing \& Crossing There is no ideal solution for differentiating a kissing from a crossing fibre, but it was observed that if the FA value is slightly increased, then one branch of the shape tends to disappear. The branch that disappears is different for both curving and kissing. The flowchart in Fig. 4 describes the process to choose between either a curving or a kissing fibre. However, this needs to be explored further and is suggested for further work.

\section{b) Measures of Algorithmic Performance}

After visually interpreting the synthetic dataset in the preceding subsection and after describing some differences between the two algorithms, in this section we wanted to quantify and analyse the results obtained. Indeed, it is of interest to both algorithm 


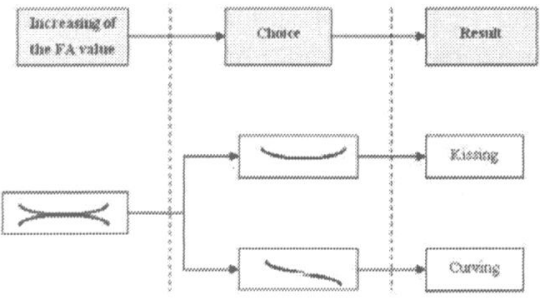

Fig. 4: Process to choose between the kissing and the curving

developers and neuroscientists to know if the two algorithms produce similar or different results for the identified fibre tracts and more importantly the extent to which the results differ.

This study was limited to two important shapes. The first one is the most basic shape, a linear fibre tract. With such a shape, computation of the pathway properties can be carried out in a simple manner. This is of particular benefit when building systems for quantitative evaluation of performance. The second shape analysed is the orthogonal crossing fibre. With this shape, an example of a crossing shape composed of two linear shapes which cross together can be examined. These two shapes were analysed for an SNR of 30 .

The steps of the process were as follows; firstly, specific fibres of the shapes were selected. This was achieved by fixing an ROI at each extremity of the shape and varying the thickness of the ROIs to generate a database. Six parameters of the chosen selections were examined. The parameters studied were the total number fibres of the selection, then for individual fibres: length, average FA value, minimum $F A$ value, average curvature and maximum angle.

Length \& FA value For both algorithms and for both shapes it can be clearly seen that the largest number of fibres have lengths of less than $50 \mathrm{~mm}$ or have an average FA lower than 0.20 . This is not unexpected as the grouping of fibres with lengths lower than $50 \mathrm{~mm}$ will include short fibres arising from surrounding noise in the image data. A similar explanation applies to fibres with an average FA lower than 0.20 . By examining distributions of the FA value higher than 0.20 , it can be observed that there is one principal concentration of FA values for the linear fibre shape example. Respectively, there are two concentrations of values for the orthogonal crossing fibre shape. It can be concluded that this concentration corresponds to the pathway. This implies that in a simple case the shape can be constructed by simply considering the FA values around this concentration.

The same analysis approach can be applied to fibre lengths. For example, it is observed that

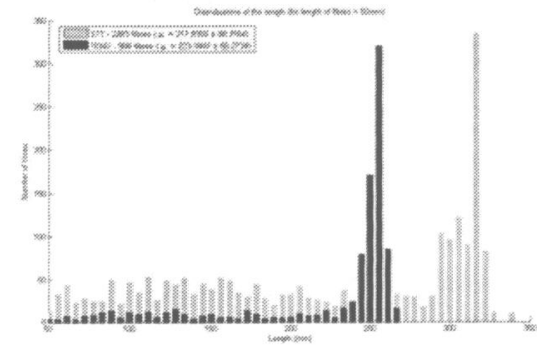

Fig. 5: Distribution of the length for fibres $>50 \mathrm{~mm}$

the largest concentration of fibre lengths for STT occurs at $320 \mathrm{~mm}$ and at $250 \mathrm{~mm}$ for the TEND. Therefore, selecting just the lengths around the peak in Fig. 5 for the chosen algorithm, guarantees a good visual representation of the linear shape.

Curvature Observations were based on the maximum angle and the average curvature. For the maximum angle, average values of approximately 15 degrees for STT and approximately 4 degrees for TEND were found. The STT value is therefore notably higher. Analysis of average curvature produces a similar result; STT values $(0.025[1 / \mathrm{mm}])$ are approximately double those of TEND $(0.012[1 / \mathrm{mm}])$. It was established that the STT algorithm results exhibit higher curvature than those from TEND. This fact is corroborated in the literature [22].

Minimum FA It was noted that the minimum FA of a fibre with TEND (e.g. linear: 0.32) was higher than with STT (e.g. linear: 0.13), but typically the average FA values were very similar (e.g. linear STT: 0.58 and TEND: 0.61).

Number of fibres In addition to these examinations it was noticed that for two ROIs there are more connecting fibres for STT than for TEND. On comparing 250 different experimental setups (location and size of ROIs) it was found that STT produces on average $60 \%$ more fibres than the TEND algorithm. It was also observed that for different thicknesses of the ROIs we get a sudden increase in the number of fibres for an ROI width of $10 \mathrm{~mm}$.

Distance Finally, with the help of the synthetic linear shape a technique was developed to compare the difference of the performance of the two algorithms. By comparing the fibres starting from a same seedpoint it can be concluded that STT and TEND produce very similar fibres, if the curvature at the extremities of the fibres identified by the STT algorithm are ignored. For the linear tract it was observed that both of the algorithms 


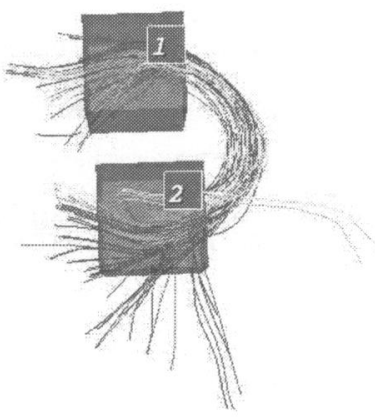

Fig. 6: Connections between two ROIs in the frontal lobes $\left(R O I_{1}:[146.7,94.3,45.5], R O I_{2}:[178.8,96.5,51.9]\right)$. Patient number 817 . In dark: TEND, in light: STT.

produce approximately straight fibres for a similar section of the pathway. For example, for the 459 fibres present in a selection ${ }^{1}$, the average distance between the pathways resulting from the STT and TEND algorithms was found to be $0.028 \mathrm{~mm}$. This demonstrates the reliability of these algorithms. While this was assessed with a straight fibre shape, it would be interesting to see how similar the results are when dealing with examples of curvature.

\section{c) Results using Real Patient Data}

Fig. 6 displays the output from this analysis framework for real patient data. Two ROIs were chosen to visualize the connections present between the two hemispheres in the frontal lobe. Firstly, it was noticed that the number of fibres is approximately the same for both algorithms (63 for STT and 66 for TEND). When comparing the distributions of length, average FA and minimum FA resulting from application of both tractography algorithms, it was found that both algorithms produced similar distribution. However, the distribution of maximum angle and the average curvature values were found to be distinct. Both the maximum angle and the curvature value were higher for STT than for TEND. This consistent with results from the synthetic dataset. As can be appreciated an understanding of the performance of the algorithms with the synthetic dataset is critical for interpretation of real patient data.

\section{Discussion}

The aim of this study was to analyse and compare different DTI tractography algorithms based on a standard framework. Using this framework, it was noted that noise is a factor impacting the quality of the eventual fibre shape. This is in line with other results reported in the literature [23]. Regarding the differences between the two algorithms, it was observed that in general the STT

\footnotetext{
${ }^{1}$ position of $\mathrm{ROI}_{1} \quad[50,236,12]$, position of $\mathrm{ROI}_{2}$ $[250,236,12]$, and thickness of the ROIs $[10,10,10]$ (data in millimeters)
}

algorithm has higher curvature than TEND. Also the importance of the FA and the length parameters was noted. Indeed with an appropriate choice of these two parameters a good representation of the fibre tract can be obtained.

A major problem for tractography algorithms is to differentiate between kissing and crossing fibres. Initially it was observed that kissing and crossing shapes give similar results. However a new approach was developed (Fig. 4) that can better detect kissing from crossing fibres in an ensemble of shapes. This analysis method hinges on the fact that we know that in our ensemble kissing or crossing fibres are present. An interesting question is how can one arrive at this conclusion without prior knowledge, in other words, how can we deal with this issue in real patient data?

A possible solution may be to further explore the FA value. Besides developing a new algorithm it would also be interesting to investigate the threshold values. For example, this could include modifying the stopping criteria for the tractography algorithm based on the distance criteria between consecutive eigenvectors. This would be an interesting approach and may provide more understanding on the utility of the FA parameter.

Concerning the FA value, an interesting observation was made: the FA values were typically very similar for both of the algorithms but the minimum FA with TEND was higher. This can be explained as fibres from STT are generally longer than fibres from TEND, so their extremities are in regions where FA is lower than elsewhere in the fibre. The minimum FA for a STT fibre will therefore have a lower value. The average $\mathrm{FA}$ value will not be greatly influenced by the minimum FA values because of the small number of voxels with low FA value. This can be validated as fibres from the same seedpoint for both TEND and STT algorithms are approximately the same in the straight sections of the fibres.

Another observation was a rapid increase in the number of fibres as a function of width. This was notable for the linear fibre shape with a width of $10 \mathrm{~mm}$. The cause of this rapid increase, and the concentration of fibres for a thickness less than $10 \mathrm{~mm}$, is difficult to interpret. It may be due to the generation of the shape, which has a thickness of almost $10 \mathrm{~mm}$, thus forcing the algorithms to build significantly more fibres in a volume of this width. Evidence to corroborate this observation is that for width less than this value, one only obtains some fibres for this shape. When we have a width of exactly $10 \mathrm{~mm}$ we obtain most of the fibres which make up the shape. For widths greater than $10 \mathrm{~mm}$ we observed fibres alongside but outside the shape. This may be one explanation of this interesting observation. 
Finally, regarding the distributions of the two major parameters, length and average $F A$ value, one can predefine the minimum and maximum value to obtain a fine outline of the pathway. Therefore these two parameters may be added to the pre-computed parameters for visualisation.

\section{FUTURE WORK}

An analysis platform was developed in conjunction with the DTI Query software, which allows us to visualise and examine fibre tracts in the brain. The next step in the development of this platform is the addition of further algorithms for tractography, such as those based on Level Set theory. Development of improved algorithms based on the two existing methods, STT and TEND, using feedback from the quantitative analysis of performance may also prove beneficial. We could also expand the study to analyse the remaining shapes from the synthetic dataset. The study could be further extended to the analysis of new fibre shapes such as two linear tracts in very close proximity. This would provide more understanding of algorithmic behaviour on simple shapes.

\section{CONCLUSION}

In this study two goals were realized to improve diffusion tensor imaging. The developed platform enables analysis of diffusion tensor image data using different tractography algorithms. The platform also allows these algorithms to be compared both quantitatively and qualitatively on real and synthetic datasets. In the absence of an anatomical gold standard it is difficult to determine which algorithm is more accurate, however the developed platform highlights differences in performance which is essential for further development of tractography algorithms.

\section{REFERENCES}

[1] P.J. Basser, J. Mattielo and D. Le Bihan. "MR Diffusion Tensor Spectroscopy and Imaging". Biophys $J$, vol. 66, pp. 259-267, 1994.

[2] C. Beaulieu. "The basis of anisotropic water diffusion in the nervous system - a technical review". NMR Bioimed. no. 15 , pp. $435-455,2002$

[3] P.J. Basser and D.K. Jones. "Diffusion-tensor MRI: theory, experimental design and data analysis - a technical review". NMR Biomed., no. 15 , pp. 456-467, 2002.

[4] R.J. Demeure. "Paramètres biophysiques fonctionnels et les IRM fonctionnelles". Louvain Med., no. 118, pp 114-128, 1999.

[5] D. Le Bihan, J.F. Mangin, C. Poupon, C. Clark, S. Pappata, N. Molko and H. Chabriat. "Diffusion Tensor Imaging: Concepts and Applications". Journal of Magnetic Resonance Imaging, no. 13, pp. 534-546, 2001.

[6] S. Mori, S. Wakana, L.M. Nagae-Poetscher and P.C.M. van Zijl. "MRI Atlas of Human White Matter". Elsevier, 2005

[7] B.A. Ardekani, A. Bappal, D. D'Angelo, M. Ashtari, T Lencz, P.R. Szeszko, P.D. Butler, D.C. Javitt, K.O. Lim,
J. Hrabe, J. Nierenberg, C.A. Branch and M.J. Hoptman. "Brain morphometry using diffusion-weighted magnetic resonance imaging: application to schizophrenia". NeuroReport, vol. 16, no. 13, pp. 1455-1459, 2005

[8] S. Pajevic C. Pierpaoli. "Color schemes to represent the orientation of anisotropic tissues from diffusion tensor data: application to white matter fiber tract mapping in the human brain". Magnetic Resonance in Medicine, no. 42, pp. 526-540, 1999.

[9] C.F. Westin, S.E. Maier, B. Khidhir, P. Everett, F.A. Jolesz and R. Kikinis. "Image Processing for Diffusion Tensor Magnetic Resonance Imaging". In Proceedings of Second Int. Conf. on Medical Image Computing and Computer assisted Interventions (MICCAI'99), pp. 441-452, 1999.

[10] P.J. Basser, S. Pajevic, C. Pierpaoli and A. Aldroubi "Fiber Tract Following in the Human Brain Using DT-MRI Data". IEICE Trans. Inf. and Syst., vol. E85-D, no. 1, pp. 15-21, 2002

[11] S. Mori and P.C.M. van Zijl. "Fiber tracking: principles and strategies - a technical review". NMR Biomed., no. 15 , pp. 468-480, 2002.

[12] C.F. Westin, S.E. Maier, H. Mamata, A. Nabavi, F.A Jolesz and R. Kikinis. "Processing and visualization for diffusion tensor MRI". Medical Image Analysis, no. 6, pp. 93-108, 2001

[13] K.H. Kim, I. Ronen, E. Formisano, R Goebel, K. Ugurbi and D.S. Kim. "Robust Fiber Tracking Method by Vector Selection Criterion in Diffusion Tensor Images". IEEE, pp. 1080-1083, 2004.

[14] P.J. Basser, S. Pajevic, C. Pierpaoli, J. Duda and A. Aldroubi. "In Vivo Fiber Tractography Using DT-MRI Data". Magnetic Resonance in Medicine, no. 44, pp. 625-632, 2000 .

[15] T.E. Conturo, N.F. Lori, T.S. Cull, E. Akbudak, A.Z. Snyder, J.S. Shimony, R.C. McKinstry, H. Burton and M.E. Raichle. "Tracking neuronal fiber pathway in the living human brain". Proc. Natl. Acad. Sci. USA 96, vol. 96, pp. 10422-10427, 1999.

[16] S. Mori, B.J. Crain, V.P. Chacko and P.C. Van Zijl. "Three-dimensional tracking of axonal projections in the brain by magnetic resonance imaging". Ann. Neurol., no. 45, pp. 265-269.

[17] M. Lazar, D.M. Weinstein, J.S. Tsuruda, K.M. Hasan K. Arfanakis, M.E. Meyerand, B. Badie, H.A. Rowley, V. Haughton, A. Field and A.L. Alecander. "White Matter Tractography Using Diffusion Tensor Deflection". Hum. Brain Mapp., no. 18, pp. 306-321, 2003.

[18] D. Weinstein, G. Kindlmann and E. Lundberg. "Tensorlines: Advection-Diffusion based Propagation through Diffusion Tensor Fields". IEEE Visualization Proceedings, pp. 249-253, 1999.

[19] http://neurology.iop.kcl.ac.uk/dtidataset/Common_DTI Dataset.htm

[20] A. Sherbondy, D. Akers, R. Mackenzie, R. Dougherty and B. Wandell. "Exploring Connectivity of the Brain's White Matter with Dynamic Queries". IEEE Trans. Visualization and Computer Graphics, vol. 11, no. 4, pp. 419:430, 2005 .

[21] D. Le Bihan and P. van Zijl. "From the diffusion coefficient to the diffusion tensor". NMR Biomed., no. 15, pp. 431$434,2002$.

[22] M. Lazar and A.L. Alexander. "An error analysis of white matter tractography methods: synthetic diffusion tensor field simulations". NeuroImage, no. 20, pp. 1140-1153, 2003.

[23] J.D. Tournier, F. Calamante, M.D. King, D.G. Gadian and A. Connelly. "Limitations and Requirements of Diffusion Tensor Fiber Tracking: An Assessment Using Simulations". Magnetic Resonance in Medicine, no. 47, pp. 701-708, 2002. 